\title{
Lower Plasma Zinc Levels in Hyperglycemic People Living with HIV in the MASH cohort
}

\author{
Alicia Sneij ${ }^{1,2}$, Adriana Campa ${ }^{1}$, Sabrina Sales Martinez ${ }^{1}$, Tiffanie Stewart ${ }^{1}$ and Marianna Baum ${ }^{1}$ \\ ${ }^{1}$ Florida International University, Florida, USA \\ ${ }^{2}$ Center for Research on US Latino HIVIAIDS and Drug Abuse (Crusada), USA
}

\begin{abstract}
Background: Zinc deficiency is prevalent in HIV and hyperglycemic patients. Antiretroviral therapy (ART) is a treatment to control HIV progression; however it increases the risk for hyperglycemia. The objective of this study was to assess the plasma zinc levels in hyperglycemic people living with HIV (PLWH).
\end{abstract}

Methods: Secondary analysis was conducted on the data from the Miami Adult Studies in HIV (MASH) cohort in Florida. Patients were categorized into hyperglycemic group (fasting blood glucose $\geq 100 \mathrm{mg} / \mathrm{dL}$ ) and normal group $(<100 \mathrm{mg} / \mathrm{dL})$.

Results: Plasma zinc status and CD4 levels were lower in the hyperglycemic group, however the difference

was not significant. There was a greater percentage of plasma zinc deficiency in the hyperglycemic group $(69 \%)$ compared to the normoglycemic group (64\%).

Discussion: Although not statistically significant, related biomarkers such as plasma zinc levels and CD4 levels were lower in the hyperglycemic group. This may be due to the role zincplays in the immune system. Due to the fact that there was a higher percentage of plasma zinc deficiency in the hyperglycemic group (69\%) compared to the normoglycemic group (64\%), it is important to monitor and manage blood glucose levels to minimize complications. Our findings along with previous findings suggest that zinc supplementation may benefit hyperglycemic PLWH.

Keywords: HIV; Antiretroviral therapy (ART); Zinc; Glucose; Hyperglycemia; Viral load; CD4 count

\section{Introduction}

Zinc deficiency is prevalent among people living with the human immunodeficiency virus (PLWH) [1-4]. This may be due to the preferential use of zinc by human immunodeficiency virus (HIV) for viral replication, altered zinc metabolism and/or inadequate dietary zinc intake [1-3]. Low plasma zinc levels directly affect the immune system because zinc is an important co-factor in the maturation of CD4 cells, which are part of the T-cell system of the immune system [5]. In addition, zinc is important for many catalytic enzymatic activity and protein/DNA synthesis [6]. Due to its importance and pervasiveness in all tissues, zinc deficiency has many adverse effects such as impairing the reconstitution of the immune function in PLWH.

In 1996, antiretroviral therapy (ART) was first used to successfully control the HIV viral load [7] and become the standard of treatment for HIV [8]. The treatment, however, has been associated with hyperglycemia and type 2 diabetes $[4,9,10]$. As a result, PLWH are at a higher risk of developing type 2 diabetes and subsequently cardiovascular disease after initiation of ART $[9,11]$.

While HIV infection is associated with numerous micronutrient deficiencies, ART has been shown to normalize plasma levels of some micronutrients after initiation [8]. However, micronutrients such as zinc, selenium and vitamin A have not been shown to increase after ART initiation [8]. Like HIV-infection, type 2 diabetes is also characterized with low plasma zinc levels $[12,13]$. This may be attributed to altered mineral metabolism and hyperzincuria [12-14]. Due to HIV infection and the association of ART to hyperglycemia and type 2 diabetes $[9,10]$, PLWH may be at higher risk of developing severe zinc deficiency.

Several studies have assessed zinc status in PLWH and in people with hyperglycemia, but not zinc status in people when both conditions are present [1-3,12-14]. Currently, research investigating the relationship of zinc status with hyperglycemia/diabetes in PLWH is lacking. The purpose of this study is to examine the relationship of plasma zinc levels and fasting blood glucose (FBG) in PLWH who are hyperglycemic/diabetic. We present data from PLWH who manifest hyperglycemic (prediabetic)/diabetic conditions and their respective zinc levels along with other related variables.

\section{Method}

A convenience sample from the Miami Adult Studies on HIV (MASH) cohort was used. The inclusion criteria were being an adult between 18-60 years of age, who had body mass index (BMI) $>18 \mathrm{~kg} /$ $\mathrm{m}^{2}$ to ensure adequate nutritional status at the beginning of the study. Participants were excluded if they were pregnant, had Hepatitis B or C, End Stage Liver Disease or any chronic inflammatory diseases such as uncontrolled diabetes. Uncontrolled diabetes is defined as FBG $\geq 140$ $\mathrm{mg} / \mathrm{dl}$ [15]. A total of 271 participants were eligible to be included in this study for analyses.

Only the baseline values of FBG, plasma zinc levels, CD4 cell count, HIV viral load, liver fibrosis and zinc intake were included in

*Corresponding author: Alicia Sneij, Florida International University, Center for Research on US Latino HIVIAIDS and Drug Abuse (CRUSADA), USA, Tel: 305978-4079; E-mail: a.sneij@gmail.com

Received November 06, 2015; Accepted February 10, 2016; Published February 15,2016

Citation: Sneij A, Campa A, Martinez SS, Stewart T, Baum M (2016) Lower Plasma Zinc Levels in Hyperglycemic People Living with HIV in the MASH cohort. J AIDS Clin Res 7: 542. doi:10.4172/2155-6113.1000542

Copyright: (C) 2016 Sneij A, et al. This is an open-access article distributed under the terms of the Creative Commons Attribution License, which permits unrestricted use, distribution, and reproduction in any medium, provided the original author and source are credited. 
the analysis. The eligible PLWH ( $n=271)$ were divided into 2 categories: hyperglycemic $(n=60)$ and normoglycemic $(n=211)$. Criteria for hyperglycemia or normal were based on the guidelines from the American Diabetes Association, which states that FBG $<100 \mathrm{mg} /$ $\mathrm{dL}$ is considered to be normal and $\geq 100 \mathrm{mg} / \mathrm{dL}$ is considered to be hyperglycemic [16].

The variables FBG, plasma zinc levels, CD4 cell count and HIV viral load were analyzed from the blood that was drawn for the parent studies. Zinc intake was obtained from a validated 24 -hour food recall which was then entered into a nutrition analyses database that provided the amount of individual nutritional components, such as zinc and total calories consumed.

Liver fibrosis is measured by FIB-4, an inexpensive and accurate tool to calculate and predict the existence of liver fibrosis. FIB-4 utilizes other variables such as age and platelet count to calculate an index that predicts the extent of liver fibrosis. FIB- 4 values $<1.45$ is known to have a negative predictive value of liver fibrosis and is indicative of a healthy and normal functioning liver; however, FIB-4 values above 3.25 has a positive predictive value of significant liver fibrosis [17].

Due to the fact that alcohol consumption inhibits intestinal zinc absorption, alcohol consumption was included in the analyses. Alcohol consumption is measured by the Alcohol Use Disorders Identification Test (AUDIT) [18] to assess any difference of alcohol consumption in the two study arms. AUDIT is a validated tool developed by the World Health Organization (WHO) to measure and assess alcohol consumption.

\section{Statistical analysis}

Descriptive statistics were used to characterize the mean and standard deviations of the continuous variables, and percent was calculated for the categorical variables. Student Independent t-test was used to determine any significant differences between the two study groups: normoglycemic $(<100 \mathrm{mg} / \mathrm{dL})$ and hyperglycemic $(\geq 100$ $\mathrm{mg} / \mathrm{dL}$ ). One-way ANOVA was used to determine any significant differences between the ethnicities/races. Finally, Pearson Correlation was used to determine any correlations between zinc intake/plasma zinc levels. $\mathrm{P}$ values $\leq 0.05$ were considered significant.

\section{Results}

Our sample population had more males than females (67\%), was generally overweight (average $\mathrm{BMI}=27.6)$, mostly African American (70\%) and were receiving ART medication (80\%). Of these characteristics, the only significant different between the 2 groups was BMI, with the hyperglycemic group having a significantly higher BMI (29.2) than the normal group (27.4) (Table 1).

Although not significant, markers of zinc deficiency $(<0.75 \mu \mathrm{g} /$ dL) [2], and disease progression (CD4 cell count) were lower in the hyperglycemic group (Table 2). In addition, the percentage of zinc deficiency was higher in the hyperglycemic group (69\%) compared to the normoglycemic group (64\%), which is equivalent to the entire sample population percentage (64\%).

The difference in liver fibrosis as measured by FIB-4 was higher in the hyperglycemic group, however, this difference was not significant $(\mathrm{P}=0.099)$. FIB- 4 values between 1.45 and 3.25 are indicative of liver fibrosis and $>3.25$ is considered to be severe liver fibrosis [17]. In our sample population, the hyperglycemic group had an average FIB4 value of $1.44 \pm 1.7$, which is approaching the parameters for liver fibrosis, while the normoglycemic group had a normal average of FIB-4 values $(1.21 \pm 0.6)$, indicative of no liver fibrosis.

Zinc intake was significantly higher in the hyperglycemic participants $(12.6 \pm 16.2$ vs. $8.5 \pm 7.1, \mathrm{P}=0.005)$, compared to the normoglycemic participants, but lost significance when adjusted

\begin{tabular}{|c|c|c|c|c|}
\hline Characteristics & Total $(n=271)$ & Hyperglycemic $(n=60)$ & Normoglycemic $(n=211)$ & P-value \\
\hline Age (years) & $45.3 \pm 7.9$ & $46.1 \pm 7.2$ & $44.6 \pm 8.3$ & 0.208 \\
\hline Male $\%$ & $67 \%$ & $62 \%$ & $66 \%$ & 0.503 \\
\hline \multicolumn{5}{|l|}{ Ethnicity \% } \\
\hline African American & $70 \%$ & $65 \%$ & $53 \%$ & 0.415 \\
\hline White & $6 \%$ & $8.3 \%$ & $5.3 \%$ & 0.388 \\
\hline Hispanic & $18 \%$ & $18.3 \%$ & $17.4 \%$ & 0.867 \\
\hline Others & $6 \%$ & $8.4 \%$ & $24.3 \%$ & --- \\
\hline $\mathrm{BMI}\left(\mathrm{kg} / \mathrm{m}^{2}\right)$ & $27.6 \pm 5.25$ & $29.2 \pm 5.7$ & $27.4 \pm 5.2$ & $0.037^{*}$ \\
\hline ART $(\mathrm{Y} / \mathrm{N}) \%$ & $80 \%$ & $88 \%$ & $82 \%$ & 0.235 \\
\hline
\end{tabular}

$p<0.05$ is considered significant and denoted by *

Table 1: Population characteristics.

\begin{tabular}{|c|c|c|c|c|}
\hline Characteristics & Total $(n=271)$ & Hyperglycemic $(n=60)$ & Normoglycemic ( $n=211)$ & P-value \\
\hline Caloric intake (kcal) & $2171 \pm 1451$ & $2608 \pm 2452$ & $2089 \pm 1019$ & 0.091 \\
\hline Zinc Intake (mg) & $9.4 \pm 9.6$ & $12.6 \pm 16.2$ & $8.5 \pm 7.1$ & $0.005^{*}$ \\
\hline Zinc intake/ 1000kcal & $4.41 \pm 3.20$ & $4.61 \pm 2.98$ & $4.25 \pm 3.02$ & 0.673 \\
\hline Plasma zinc level $(\mu \mathrm{g} / \mathrm{dL})$ & $0.73 \pm 0.3$ & $0.70 \pm 0.2$ & $0.74 \pm 0.3$ & 0.360 \\
\hline Zinc deficient (\%) & $64 \%$ & $69 \%$ & $64 \%$ & ---- \\
\hline CD4 cell count (cells $/ \mathrm{mL}^{3}$ ) & $497.3 \pm 357.01$ & $417.8 \pm 439.40$ & $436.8 \pm 344.70$ & 0.693 \\
\hline Viral load $\left(\log _{10}\right)$ & $2.70 \pm 1.3$ & $2.72 \pm 1.3$ & $2.73 \pm 1.2$ & 0.959 \\
\hline Albumin (mg/dL) & $4.25 \pm 0.3$ & $4.3 \pm 0.4$ & $4.23 \pm 0.3$ & 0.179 \\
\hline FIB-4 & $1.32 \pm 1.1$ & $1.44 \pm 1.7$ & $1.21 \pm 0.6$ & 0.099 \\
\hline AUDIT Score & $9.45 \pm 10.1$ & $9.61 \pm 10.2$ & $9.8 \pm 10.1$ & 0.900 \\
\hline
\end{tabular}

$p<0.05$ is considered significant and denoted by * 
by intake in $\mathrm{g} / 1000 \mathrm{kcal}$. A Pearson correlation showed a negative correlation $(\mathrm{r}=-0.87 ; \mathrm{p}=0.140)$ between zinc intake and plasma zinc levels in the sample population. There was a positive correlation in the hyperglycemic group $(\mathrm{r}=0.24 ; \mathrm{p}=0.861)$ and a negative correlation in the normoglycemic group $(\mathrm{r}=-0.117 ; \mathrm{p}=0.076)$ between zinc intake and plasma zinc levels.

\section{Discussion}

The results obtained in this secondary analysis of data confirm findings from previous studies on the prevalence of zinc deficiency in HIV and hyperglycemic/diabetic populations $[2,13]$. Findings are also consistent with the literature on obesity that shows that higher BMI is a risk factor for both hyperglycemia and liver fibrosis [19,20]. Both study groups were zinc deficient $(<0.75 \mu \mathrm{g} / \mathrm{dL})$ with the hyperglycemic group being more deficient $(0.70 \pm 0.2 \mu \mathrm{g} / \mathrm{dL})$. While this difference was not significant, it is important to understand that uncontrolled diabetes was excluded in the original data repository, thus preventing access to a large portion of hyperglycemic/diabetic participants. As a result, the hyperglycemic sample used in the secondary analysis is not necessarily representative of the HIV hyperglycemic population, and certainly not of the most at-risk population.

The lack of correlation between zinc intake and plasma zinc levels confirms that there are probably more than one factor affecting zinc absorption, utilization and metabolism. Previous studies have shown variable absorption rates related to zinc status and zinc intake. This might signify that the body compensates for inadequate zinc status and intake $[7,21,22]$. Considering that this population had more men than women (67\%), they should be consuming zinc at the recommended dietary allowances (RDA) levels, which is $11 \mathrm{mg} /$ day for men and $8 \mathrm{mg} /$ day for women [23]. We observed that the hyperglycemic group consumed adequate amounts of zinc $(\sim 12 \mathrm{mg})$, however the normoglycemics reported a mean zinc intake that was inadequate ( $\sim 8 \mathrm{mg}$ ) but their plasma levels were higher. The negative correlation between zinc intake and status confirms previous studies suggesting compensation in bioavailability for inadequate zinc intake $[7,21,22]$.

Although the difference is not significant, CD4 levels were lower in the hyperglycemic group (417.8) compared to the normal group (436.8). This is in conjunction with lower plasma zinc levels $(0.70 \pm$ 0.2 ) and a higher proportion of zinc deficient subjects (69\%) in the hyperglycemic group. This is important to consider because zinc is a necessary cofactor for the maturation of $\mathrm{CD} 4 \mathrm{~T}$ cells which play a vital role in the immune system. When cell counts drop to $<200$ cells/ $\mathrm{mm}^{3}$, it is considered to be immunological failure [2] and a diagnosis of Acquired Immunodeficiency Syndrome (AIDS) [24]. A study conducted by Baum et al. [2] found that supplementation of zinc to HIV-infected adults for 18 months resulted in a 4 -fold decrease in the likelihood of developing immunological failure. As a result, they are less susceptible to opportunistic infections and have a decreased risk of mortality [2].

The importance of maintaining adequate plasma zinc levels for immune function is evident especially in PLWH, due to the observed deficiency of zinc in this particular population [2,25]. Zinc deficiency is also observed in hyperglycemic conditions such as diabetes [12,13], which may be worsened when co-occurring with HIV infection. Due to the fact that ART medication increases the risk of developing diabetes in PLWH, type 2 diabetes is prevalent in the HIV population and may pose an additional risk for disease progression and complications. Our results have not found significant difference in plasma zinc levels between the hyperglycemic and normoglycemic groups, but this may be due to the small sample size and the fact that uncontrolled diabetes was excluded from our data repository. Although our findings do not show significance, it is noteworthy to mention that the plasma zinc levels were lower in the hyperglycemic group despite adequate zinc intake. In addition, there was a higher percentage of zinc deficiency in the hyperglycemic group (69\%) compared to the normoglycemic group (64\%).

\section{Limitations}

The sample size is limited by the availability of the data repository, and the direction and effect size of the analyses indicate that a larger sample size will be needed to assess the relationship between hyperglycemia and zinc status.

This was a secondary analysis of data collected for other purposes, which limits the availability of other variables, such as acute phase reaction, which is needed to adjust the relationship between intake and plasma zinc levels. In addition, uncontrolled diabetics were excluded from the parent study, thus reducing our sample size and the ability to observe the effects of extreme hyperglycemic values. We presume that our findings would approach significance if our sample size was larger and cases of uncontrolled diabetes were included in our analyses.

We utilized 24-hour food recalls to estimate zinc intake, which is dependent on the memory and accuracy of the participants providing the information. Finally, acute phase reactants, such as C-reactive protein, were not measured and they may be an important covariate since zinc is sequestered in all cells under acute inflammation producing a temporary false deficiency status.

\section{Conclusions}

Previous studies have shown altered glucose and lipid metabolism in HIV patients taking ART. This has resulted in increased levels of FBG and increased cardiovascular risk factors such as diabetes $[4,10,11]$. While ART has been shown to normalize other micronutrient plasma levels, zinc levels remains deficient [8]. Our findings confirm that plasma zinc is deficient in HIV infection and hyperglycemia and it is slightly more deficient when both conditions are present, but the difference is not significant. We also found a higher percentage of plasma zinc deficient subjects in the hyperglycemic group (69\%) compared to the normoglycemic group (64\%). Moreover, CD4 cells were slightly lower in the hyperglycemic group but the difference was not significant. Further research in this particular field is warranted to minimize the complications associated with HIV and ART associated-hyperglycemia. PLWH are encouraged to monitor their FBG and plasma zinc status after initiating ART to prevent complications associated with these conditions.

\section{Acknowledgment}

The authors wish to thank all the participants and staff at the FIU-Borinquen clinic who provided their time and effort during data collection and analyses and who made this research project possible.

\section{Conflicts of Interest}

The data repository from the MASH cohort was funded by the "Alcohol \& Antiretrovirals in HIV Infection, Oxidative Stress and Liver Disease" (Grant \#: R01AA018011) and "HIV and HIV/HCV-Infection, Disease Progression, Oxidative Stress and Antioxidants" (Grant \#: R01DA023405).

This work was also conducted under the NIH funded CRUSADA grant (Grant \# P20MD002288) 
Citation: Sneij A, Campa A, Martinez SS, Stewart T, Baum M (2016) Lower Plasma Zinc Levels in Hyperglycemic People Living with HIV in the MASH cohort. J AIDS Clin Res 7: 542. doi:10.4172/2155-6113.1000542

Page 4 of 4

\section{References}

1. Visser ME, Maartens G, Kossew G, Hussey GD (2003) Plasma vitamin A and zinc levels in HIV-infected adults in Cape Town, South Africa. Br J Nutr 89: 475-482.

2. Baum MK, Lai S, Sales S, Page JB, Campa A (2010) Randomized, controlled clinical trial of zinc supplementation to prevent immunological failure in HIVinfected adults. Clin Infect Dis 50: 1653-1660.

3. Baum MK, Campa A, Lai S, Lai H, Page JB (2003) Zinc status in human immunodeficiency virus type 1 infection and illicit drug use. Clin Infect Dis 37: S117-123.

4. Wand H, Calmy A, Carey DL, Samaras K, Carr A, et al. (2007) Metabolic syndrome, cardiovascular disease and type 2 diabetes mellitus after initiation of antiretroviral therapy in HIV infection. AIDS 21: 2445-2453.

5. Kahmann L, Uciechowski P, Warmuth S, Malavolta M, Mocchegiani E, et al. (2006) Effect of improved zinc status on T helper cell activation and TH1/TH2 ratio in healthy elderly individuals. Biogerontology 7: 429-435.

6. Beyersmann D, Haase $H(2001)$ Functions of zinc in signaling, proliferation and differentiation of mammalian cells. Biometals 14: 331-341.

7. Ellie W, Sharon W (2008) Understanding Nutrition (11th edn.). Thomson Wadsworth 452.

8. Drain PK, Kupka R, Mugusi F, Fawzi WW (2007) Micronutrients in HIV-positive persons receiving highly active antiretroviral therapy. Am J Clin Nutr 85: 333345

9. De Wit S, Sabin CA, Weber R, Worm SW, Reiss P, et al. (2008) Incidence and risk factors for new-onset diabetes in HIV-infected patients: the Data Collection on Adverse Events of Anti-HIV Drugs (D:A:D) study. Diabetes Care 31: 12241229.

10. Brown TT, Cole SR, Li X, Kingsley LA, Palella FJ, et al. (2005) Antiretroviral therapy and the prevalence and incidence of diabetes mellitus in the multicenter AIDS cohort study. Arch Intern Med 165: 1179-1184.

11. Fields-Gardner C, Campa A, American Dietetics Association (2010) Position of the American Dietetic Nutrition Intervention and Human Immunodeficiency Virus Infection. Journal of the American Dietetic Association 110: 1105-1119.

12. Al-Maroof RA, Al-Sharbatti SS (2006) Serum zinc levels in diabetic patients and effect of zinc supplementation on glycemic control of type 2 diabetics. Saudi Med J 27: 344-350.

13. Anetor JI, Senjobi A, Ajose OA, Agbedana EO (2002) Decreased serum magnesium and zinc levels: atherogenic implications in type-2 diabetes mellitus in Nigerians. Nutr Health 16: 291-300.

14. Ferdousi S, Mia AR, Mahzabeen L, Nath RK, Mollah FH (2013) Serum zinc and magnesium level in newly diagnosed type-2 diabetic subjects. Mymensingh Med J 22: 552-556.

15. Chakrabarti A, Das A, Mandal J, Shivaprakash MR, George VK, et al. (2006) The rising trend of invasive zygomycosis in patients with uncontrolled diabetes mellitus. Med Mycol 44: 335-342.

16. Bansal N (2015) Prediabetes diagnosis and treatment: A review. World $J$ Diabetes 6: 296-303.

17. Vallet-Pichard A, Mallet V, Nalpas B, Verkarre V, Nalpas A, et al. (2007) FIB-4 an inexpensive and accurate marker of fibrosis in HCV infection. comparison with liver biopsy and fibrotest. Hepatology 46: 32-36.

18. World Health Organization (2012). Alcohol Use Disorder Identification Test (AUDIT).

19. Cosio, FG, Kudva Y, van der Velde M, Larson TS, Textor SC et al. (2005) New onset hyperglycemia and diabetes are associated with increased cardiovascular risk after kidney transplantation. Kidney International 67.

20. Angulo P, Keach JC, Batts KP, Lindor KD (1999) Independent predictors of liver fibrosis in patients with nonalcoholic steatohepatitis. Hepatology 30: 13561362.

21. Lönnerdal B (2000) Dietary factors influencing zinc absorption. J Nutr 130 1378S-1383S

22. Krebs NF (2000) Overview of zinc absorption and excretion in the human gastrointestinal tract. J Nutr 130: 1374S-1377S.

23. Health, National Institutes of Zinc (2013) Office of Dietary Supplements.

24. Longosz AF, Mehta SH, Kirk GD, Margolick JB, Brown J, et al. (2014) Incorrect identification of recent HIV infection in adults in the United States using a limiting-antigen avidity assay. AIDS 28: 1227-1232.

25. Baum MK, Shor-Posner G, Campa A (2000) Zinc status in human immunodeficiency virus infection. J Nutr 130: 1421S-1423S. 